\title{
Diagnosis and Treatment of an Undifferentiated Embryonal Sarcoma of the Liver in a 37-Week Pregnant Woman: A Case Report
}

\author{
Tran Manh Hung (D) \\ Tran Que Son (iD 1,2 \\ Tran Thi Phuong ${ }^{3}$ \\ Hoang Van Minh (D) ${ }^{4}$ \\ 'Department of Surgery, Bach Mai \\ Hospital, Hanoi, Vietnam; ${ }^{2}$ Hanoi Medical \\ University, Hanoi, Vietnam; ${ }^{3}$ Department \\ of Epidemiology, Thai Binh University of \\ Medicine and Pharmacy, Thaibinh, \\ Vietnam; ${ }^{4}$ Hanoi University of Public \\ Health, Hanoi, Vietnam
}

Correspondence: Tran Manh Hung Department of Surgery, Bach Mai Hospital, Hanoi, Vietnam

Email tranmanhhungngoaibm@gmail.com
Purpose: Undifferentiated embryonal sarcoma of the liver (UESL) is a rare malignant lesion in adults. Here, we present an extremely rare case of UESL in a pregnant woman. However, this was the first case, wherein one surgery covered all the procedures needed to be performed, including delivery of the neonate, hysterectomy, and resection of the left liver lobe to ensure the safety of both the mother and the newborn.

Case Presentation: A 32-year-old woman at 37-weeks age of gestation was admitted at Bach Mai Hospital (Hanoi, Vietnam) due to epigastric pain, chest pain, and breathing difficulty. Blood tests showed an increased total alpha-fetoprotein level of $1085 \mathrm{ng} / \mathrm{mL}$. Fetal ultrasound showed a fetal heart rate of 127 beats/minute, biparietal diameter of $92 \mathrm{~mm}$, femur length of $70 \mathrm{~mm}$, and fetal weight of $3287 \mathrm{~g}$. Magnetic resonance imaging indicated a large tumor $(12 \times 14 \times 18 \mathrm{~mm})$ in the left liver, with organ signals increased on T2-weighted and decreased on T1-weighted. The mass was lobulated, developed along a downward pattern, and did not invade the portal vein. The surgeries performed were cesarean section, hysterectomy due to the inelasticity of the uterus, and left hepatectomy to ensure the safety of both the mother and newborn. The operation was performed safely and without any complications, and the patient was discharged from the hospital after 10 days.

Conclusion: This case report with distinct characteristics has been the first case ever reported in Vietnam, as well as in the literature. We believe that this case report will be useful for the clinical evidence pool and for educational purposes.

Keywords: hepatic tumor, undifferentiated embryonal sarcoma, hepatic resection, cesarean section, hysterectomy, case report

\section{Introduction}

Undifferentiated embryonal sarcoma of the liver (UESL) is a very rare form of liver cancer in adults. ${ }^{1}$ Until now, there have been just over 60 clinical cases reported in the literature. ${ }^{2}$ UESL tends to have non-specific clinical and imaging diagnostic features, which can lead to misdiagnosis of the tumor origin; hence, delays in choosing appropriate treatment methods. Surgery is still the first choice of treatment, and radical surgery should be performed in all cases. In this paper, we reported a special case of UESL in a 37-weeks pregnant woman. This form of malignant tumor is rarely found in pregnant women, and this was the first case to perform surgery that involved all procedures of delivering the neonate, hysterectomy, and left liver resection in one operation to ensure the 
safety of both the mother and newborn. We believe that this case report will be useful for the clinical evidence pool and for educational purposes.

\section{Case Presentation}

The patient was a 32-year-old woman who was 37-week pregnant (third time) and was admitted to the Bach Mai Hospital (Hanoi, Vietnam) due to epigastric pain, chest pain, and difficulty of breathing. Physical examination showed that the size of the uterus was appropriate for a gestational age of 37 weeks, with normal fetal heartbeat and no contractions. Additionally, a non-mobile, painless, $20-\mathrm{cm}$ tumor was detected in the left liver, which was present since the previous year $(<3 \mathrm{~cm})$ but was not treated due to the patient's unwary of the importance of early treatment and poor economic conditions.

Blood tests showed an increase in total alphafetoprotein (AFP) of $1085 \mathrm{ng} / \mathrm{mL}$, and other indicators were present at normal levels (AFP, L3 0.3\%; Prothrombin induced by the absence of vitamin $\mathrm{K}$ or antagonist-II (PIVKA-II) $32 \mathrm{mAU} / \mathrm{mL}$; red blood cells $3.76 \mathrm{~T} / \mathrm{L}$; white blood cells $9.6 \mathrm{~g} / \mathrm{L}$; platelet count, 184 $\mathrm{g} / \mathrm{L}$; prothrombin, $109 \%$ urea $3.2 \mathrm{mmol} / \mathrm{L}$; creatinine, 51 $\mu \mathrm{mol} / \mathrm{L}$; total protein, $69.1 \mathrm{~g} / \mathrm{L}$; albumin, $35.4 \mathrm{~g} / \mathrm{L}$; total bilirubin, $9.6 \mu \mathrm{mol} / \mathrm{L}$; serum AST, $35 \mathrm{U} / \mathrm{L}$; serum ALT, 11 $\mathrm{U} / \mathrm{L}$ ). Magnetic resonance imaging (MRI) indicated that a large tumor $(12 \times 14 \times 18 \mathrm{~mm})$ in the left liver, with organ signals increased for T2-weighted (Figure 1) and decreased for T1-weighted (Figures 2 and 3). The mass

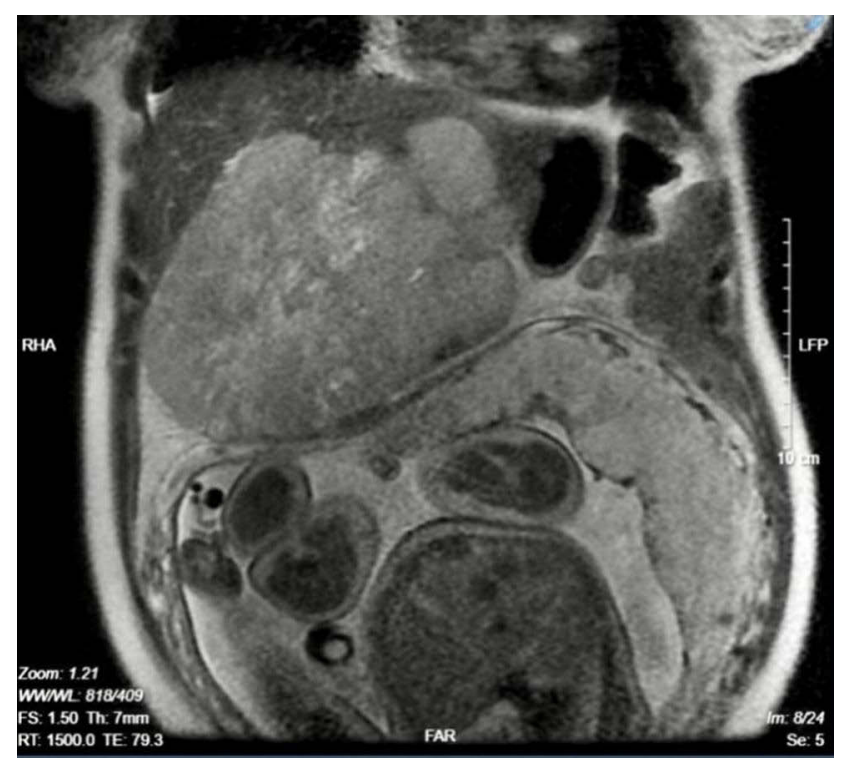

Figure I Liver tumor on T2.

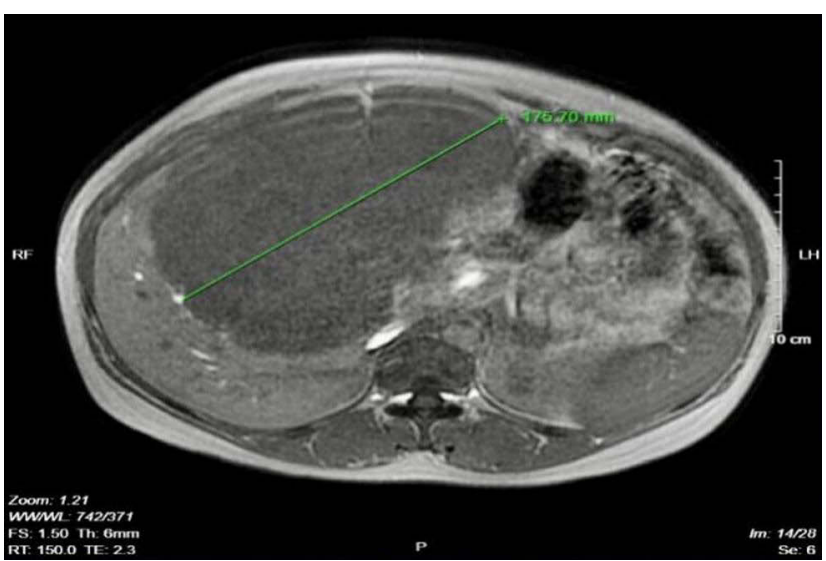

Figure 2 Liver tumor on TI in-phase.

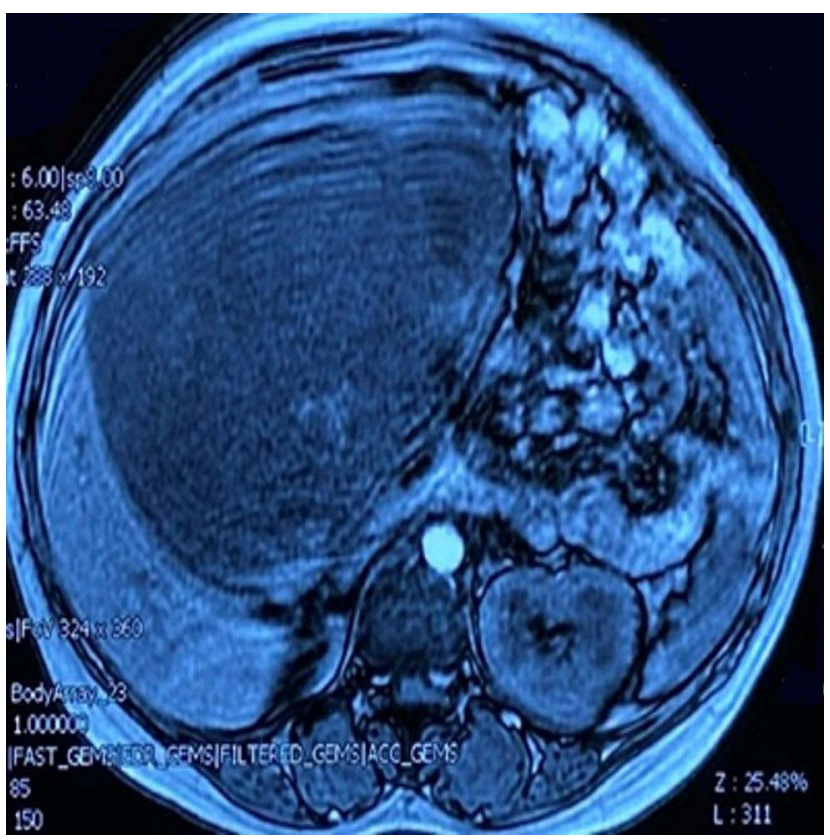

Figure 3 Liver tumor on TI out-of -phase.

had a lobe, developed along a downward pattern, and did not invade the portal vein. However, it compressed the lower part of the uterus, gallbladder, and pancreas. There was no free abdominal fluid, and the right liver did not show any tumors.

Fetal ultrasound showed a fetal heart of 127 times/ minute, bipolar diameter of $92 \mathrm{~mm}$, femoral length of $70 \mathrm{~mm}$, head-to-toe length of $48.5 \mathrm{~mm}$, head circumference of $329 \mathrm{~mm}$, waist circumference of $328 \mathrm{~mm}$, and fetal weight of $3287 \mathrm{~g}$. The amniotic fluid was normal, the placenta was attached to the back of the uterus, and there were no fetal or uterine abnormalities noted. 
This case was consulted by related specialists of surgery, obstetrics, resuscitation, radiology, nuclear medicine, oncology, pathology, pediatrics, and gastroenterology. As discussed, the expected surgical methods for this patient were a cesarean section, hysterectomy if the uterus had a poor contraction, and left hepatectomy. These were performed consecutively.

The middle linear alba below the navel was opened to deliver the neonate, weighing $3100 \mathrm{~g}$. As for the mother, because the uterus was bleeding and contracting poorly, partial hysterectomy was performed. Subsequently, the surgical team extended the abdominal incision to the sternum. The abdomen was dry with no signs of metastasis to the peritoneum, and the right liver was soft with a pink color. The large tumor developed in the left liver lobe, encroaching on the inferior part of segment IV, with downward convex development which compressed the stomach and the transverse colon. Complete hepatic pedicle control was performed once in $15 \mathrm{~min}$. A left hepatectomy including the inferior segments II, III, and IV was performed according to the Ton That Tung method. ${ }^{15}$ The liver veins and left liver pedicles were resected with a blood vessel stapler of $35 \mathrm{~mm}$. The total weight of the resected tumor was $3000 \mathrm{~g}$. Due to the $350 \mathrm{~mL}$ blood loss during the hepatectomy, $700 \mathrm{~mL}$ of red blood cells and $250 \mathrm{~mL}$ of fresh plasma was transfused during surgery. The surgery was performed safely and without any complications within 120 minutes in June 2020.

Postoperatively, the patient was awake and was able to ingest liquid food after 48 hours. Her skin and mucosa remained pink, the abdomen was soft, and the incision was dry and clean. Douglas drainage was withdrawn on the third postoperative day, and subhepatic drainage was removed on the fifth postoperative day. Blood tests on the first, third, and fifth postoperative days showed normal results. Biochemical and hematology tests on the third and fifth postoperative days were also within the normal ranges. The patient was discharged from the hospital after 10 days. One month after surgery, the patient received six courses of chemotherapy (one course lasted 3 weeks). Six months after surgery, chemotherapy was completed, and the patient was completely healthy.

The patient's patho-biological characteristics showed that the left liver had dimensions of $20 \times 16 \times 12 \mathrm{~mm}$, and a tumor $(16 \times 13 \times 12 \mathrm{~mm})$ approximately $1 \mathrm{~cm}$ from the liver section. The cross-section was soft and gray, and the necrosis was wide at $70 \%$. The tumor structure consisted of a polyhedron and elongated rhombohedral cells with coarse chromatin and multiple monstrous nuclei. Tumor cells were in clumps or rafts and separated by thinwalled blood vessels. Invasive vascular injury was not observed. The remaining resected areas of the liver did not contain any more of the tumor (Figure 4). Immunohistochemical staining showed positive CD34 (Figure 5), negative CD117, negative Dog-1, negative glypican, positive CK (Figure 6), and CD99 (Figure 7).

\section{Discussion}

Stocker and Ishak first detected UESL in 1978. To date, very few cases of UESL in adults have been reported in the literature. ${ }^{1-5}$ Undifferentiated mesenchymal carcinoma

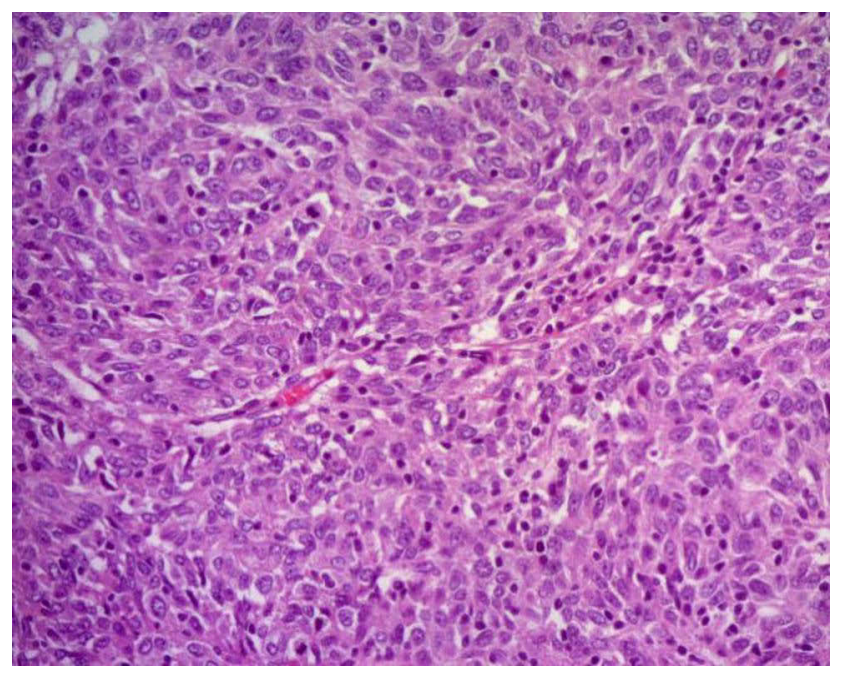

Figure 4 Tumor histopathology.

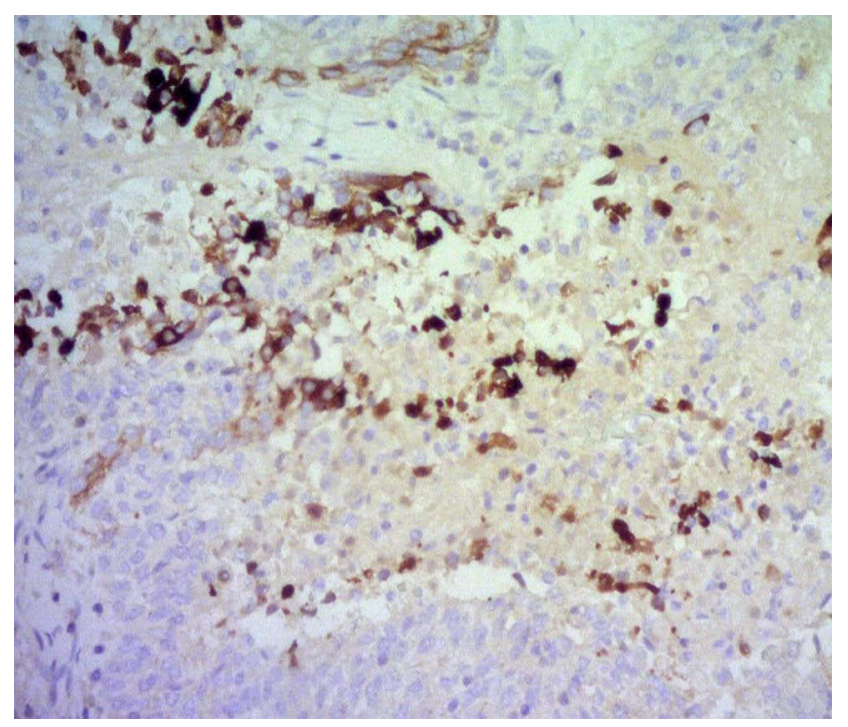

Figure 5 Positive CD34. Monoclonal Mouse Anti-Human CD34 Class II Clone QBEnd 10 DK-2600 Glostrup, Denmark. 


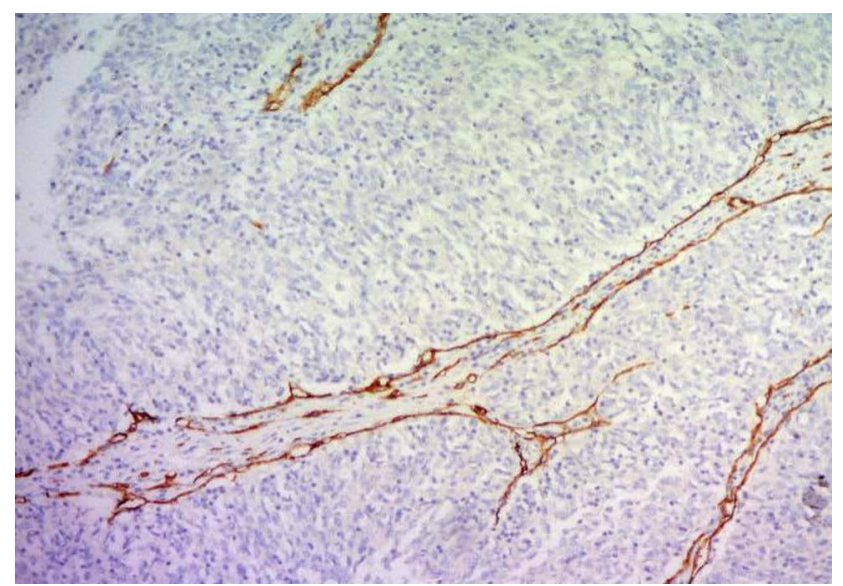

Figure 6 Immunohistochemistry CK (+). Monoclonal Mouse Anti-Human Cytokeratin Clone MNF II6 DK-2600 Glostrup, Denmark.

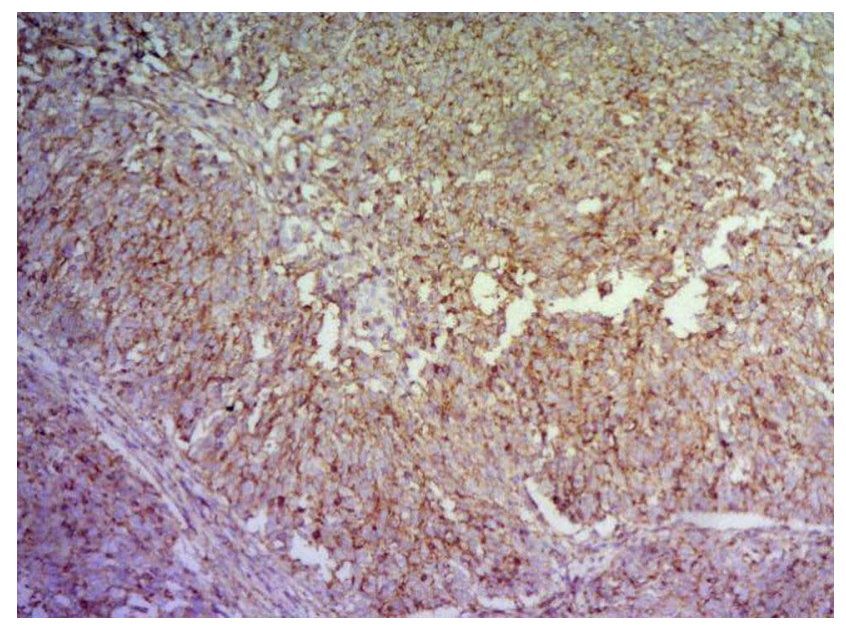

Figure 7 Positive CD99. Rabbit Monoclonal Primary Antibody EPR3097Y RabMAb.

can occur anywhere in the liver but usually develops on the right side. Clinical manifestation of UESL include unintentional weight loss, loss of appetite, chest pain, and dyspnea. The tumor could also be detected during selfexamination. An ultrasound scan may reveal a large tumor size with high fluid volume, which can easily be mistaken for an abscess. Computed tomography may show inconsistent images of the tumor, hypochromic, and abnormal contrast enhancement. MRI may be used to detect signs of vascular invasion to better predict the possibility of surgical resection. Immunohistochemical staining can also be performed to ensure a definitive and differential diagnosis of UESL from other conditions. These diagnostic techniques must be conducted based on the fact that only one biomarker cannot warrant a diagnosis, but multiple biomarkers should be examined to distinguish from various types of tumors. ${ }^{4,6,8,9}$ UESL has a poor prognosis with
5 -year survival rate of less than $37 \% .{ }^{5,9}$ In one study, the median survival probability after 5 years among 308 patients was $65.8 \%$, which increased to $70 \%$ with hepatectomy and to $78.9 \%$ with liver transplant, whereas survival was decreased to only $6.6 \%$ in those who did not undergo surgery. In addition, liver resection combined with adjuvant chemotherapy prolongs survival. ${ }^{4}$ The chemotherapeutic drugs used are vincristine, actinomycin D, doxorubicin, cyclophosphamide, tiropramide, and cisplatin. $^{2,4,10}$

UESL in pregnant women is very rare, and all these few case reports appear to have different characteristics. There were two cases in which patients underwent hepatectomy and had a relapse and metastasis of UESL prior to being pregnant. ${ }^{11,12}$ The first case was of a first-time pregnant woman with preeclampsia. She was diagnosed with UESL in the left liver, which had invaded the interior vena cava and the right side of the heart. ${ }^{13}$ Another pregnant patient presented with abdominal pain and dyspnea and was found to have a large UESL that invaded almost her entire liver, with multiple scattered metastases in the liver. ${ }^{14}$ In a case reported by Jiang et al in $2021,{ }^{15}$ a patient was diagnosed with UESL by ultrasound-guided right liver biopsy at 14th week of pregnancy. She underwent right hepatectomy at the 15th week of pregnancy, with a focus on monitoring and preserving the fetus. In our case, the patient also had distinct characteristics, and to the best of our knowledge, this case is the first to be reported in Vietnam and in the literature. It is worth noting that this was a very large tumor in a 37 -week pregnant woman of unknown origin before surgery. We had to come up with a solution of a cesarean section, hysterectomy, and left hepatectomy within one operation to ensure the safety of both the mother and newborn. The postoperative pathology results also confirmed UESL.

AFP is a protein produced by the yolk sac and the fetal liver. It is present in the amniotic fluid that crosses the placenta into the bloodstream of the mother. AFP is often used to diagnose primary liver cancer, testicular cancer, ovarian cancer, fetal malformations, or multiple pregnancies. In our case, a pregnant woman with a very large liver tumor had an AFP level increase of $1085 \mathrm{ng} / \mathrm{mL}$, but AFPL3 was $0.3 \%$ and PIKA-II was $32 \mathrm{mAU} / \mathrm{mL}$. We believe that this was an indicator of a malignancy in the liver and not a fetal malformation because the pregnancy progressed normally, and the ultrasound did not detect any abnormalities in the fetus. In fact, the newborn was completely healthy and developed normally. The pathology result of 
the mother's liver tumor revealed a UESL. Thus, abnormal AFP levels in pregnant women may also signal maternal malignancy, which has not been mentioned in previous reports. ${ }^{14,15}$ In this case, the unfortunate thing is that a tumor less than $3 \mathrm{~cm}$ in the left liver was detected at a local hospital 1 year ago, but the patient did not go to a tertiary hospital for early diagnosis and treatment due to unwary and poor economic conditions. She became pregnant for the 3rd time, with no maternity follow-up, and no periodic check for the liver tumor. Therefore, the tumor enlarged quickly during the pregnancy period. This is an observable fact in developing countries.

Normal delivery is still the first choice of obstetrician because it is the best and most natural birth method for mothers and babies. Although cesarean section is faster than normal delivery, it could have acute or long-term consequences and complications for the mother. In our case, although the 37-week pregnant woman had no signs of labor, two previous normal deliveries, and no signs of difficult labor from the fetus, we still performed an elective cesarean section because she had a very large tumor in the liver with aggressive clinical manifestations, and the risk of tumor rupture is life-threatening for both the mother and baby. This is a reasonable and safe indication for this case.

These tumors have atypical clinical symptoms and imaging features, most of which cannot be diagnosed before surgery, especially with a large left liver tumor being at risk of rupture, causing acute bleeding or metastasis. Hence, treatment requires surgical intervention. However, this patient was pregnant for the 3rd time (the first two children were healthy), and the fetus developed well. Many opinions among specialists have been discussed. The first option was that only the cesarean section should be performed, the uterus was preserved, and hepatectomy was performed after 1 month. The second option was cesarean section, preserving the uterus, and then hepatectomy in one operation. The third option was a cesarean section, hysterectomy if the uterus had a poor contraction, and left hepatectomy in one operation. In the first option, in terms of obstetrics, it could be safer for the fetus and mother because the patient underwent a cesarean section only, so the risk of blood loss was low. However, the patient must undergo further surgery for hepatectomy. During that time, the tumor may have ruptured or metastasized. Therefore, most of our team member did not agree with this option. In our opinion, if the patient had signs of fetal poisoning, placental abruption, blood clotting disorders, or severe combined internal disease (such as heart failure and kidney failure), the first option should be chosen to ensure the safety of both the mother and child. In the second and third options, there was a consensus that a cesarean section should be combined with hepatectomy. However, if the uterus was preserved after the cesarean section but did not contract well, the patient would be at risk of bleeding from the uterine opening incision or endometrium. This woman gave birth to her third child and had two first healthy children. Therefore, the hysterectomy plan if the uterus had a poor contraction, which preserved the adnexa uteri (to minimize the risk of bleeding from the uterus), combined with left hepatectomy in one operation, was agreed upon.

\section{Conclusion}

Surgery for UESL in a 37-week pregnant woman was a special case that was successful. Both the mother and newborn were safe after the surgery. The results showed that the indication of the cesarean section and hepatectomy at once, in this case, has been an optimal choice because it ensured the safety of both the mother and child. The hepatectomy technique using Ton That Tung's method was safe and effective, because the huge liver tumor caused pressure on the liver pedicles and surrounding organs, and it was difficult to reach the left liver pedicles. A similar clinical situation should only be performed in hospitals that can perform surgical and obstetric techniques.

\section{Abbreviations}

MRI, magnetic resonance imaging; UESL, undifferentiated embryonal sarcoma of the live; AFP, alphafetoprotein; PIVKA-II, prothrombin induced by the absence of vitamin $\mathrm{K}$ or antagonist-II.

\section{Data Sharing Statement}

This case report contains clinical data from the medical records in the Bach Mai hospital. Additional information is available from the first author upon reasonable request.

\section{Ethics Approval and Informed Consent}

Writing and publishing this case report was approved by Bach Mai hospital. 


\section{Consent for Publication}

Written informed consent was obtained from the patient for publication of this case report and the accompanying images. A copy of the written consent is available upon request.

\section{Acknowledgment}

The authors would like to thank our patients and her family for their support.

\section{Author Contributions}

All authors contributed to data analysis, drafting or revising the article, gave final approval of the version to be published, agreed to the submitted journal, and agreed to be accountable for all aspects of the work.

\section{Funding}

This case report involved no source of funding for any of the authors.

\section{Disclosure}

The authors declare that they have no competing interest.

\section{References}

1. Schepelew D, Reese T, Horling K, Frenzel C, Oldhafer KJ. Undifferentiated embryonal sarcoma of the liver treated with associating liver partition and portal vein ligation for staged hepatectomy in a young adult: a case report. Int J Surg Case Rep. 2020;66:221-227. doi:10.1016/j.ijscr.2019.11.052

2. Pinamonti M, Vittone F, Ghiglione F, Borasi A, Silvestri S, Coverlizza S. Unexpected liver embryonal sarcoma in the adult: diagnosis and treatment. Case Rep Surg. 2018;2018:1-5. doi:10.1155/ 2018/8362012

3. Stocker JT, Ishak KG. Undifferentiated (embryonal) sarcoma of the liver: report of 31 cases. Cancer. 1978;42(1):336-348. doi:10.1002/ 1097-0142(197807)42:1<336::aid-cncr2820420151>3.0.co;2-v
4. Wu Z, Wei Y, Cai Z, Zhou Y. Long-term survival outcomes of undifferentiated embryonal sarcoma of the liver: a pooled analysis of 308 patients. ANZ J Surg. 2020;90(9):1615-1620. doi:10.1111/ans.15684

5. Cao Q, Ye Z, Chen S, Niu L, Li S, Liu F. Undifferentiated embryonal sarcoma of liver: a multi-institutional experience with 9 cases. Int J Clin Exp Pathol. 2014;7(12):8647-8656. PubMed PMCID: PMC4313983. PMID25674229.

6. Almogy G, Pappo O, Gips M, Lieberman S, Edden Y, Eid A. Improved survival with surgery and systemic chemotherapy for undifferentiated embryonal sarcoma of the liver. Isr Med Assoc J. 2005;7(10):672-673. PubMed PMID16259353.

7. Kiani B, Ferrell LD, Qualman S, Frankel WL. Immunohistochemical analysis of embryonal sarcoma of the liver. Appl Immunohistochem Mol Morphol. 2006;14(2):193-197. doi:10.1097/01.pai.0000173052. 37673.95

8. Putra J, Ornvold K. Undifferentiated embryonal sarcoma of the liver: a concise review. Arch Pathol Lab Med. 2015;139(2):269-273. doi:10.5858/arpa.2013-0463-RS

9. Baron PW, Majlessipour F, Bedros A, et al. Undifferentiated embryonal sarcoma of the liver successfully treated with chemotherapy and liver resection. J Gastrointest Surg. 2007;11(1):73-75. doi:10.1007/ s11605-006-0044-4

10. Shufaro Y, Uzieli B, Pappo O, Abramov Y. Pregnancy and delivery in a patient with metastatic embryonal sarcoma of the liver. Obstet Gynecol. 2002;99(5 Pt 2):951-953.

11. McCarthy FP, Harris M, Kornman L. Management of undifferentiated embryonal sarcoma of the liver in pregnancy. Obstet Gynecol. 2007;109(2 Pt 2):558-560. doi:10.1097/01.AOG.00002 52833.73033 .44

12. Akerboom-Straberger BM, Lotgering FK. Embryonal sarcoma of the liver in pregnancy, associated with HELLP syndrome. Am J Obstet Gynecol. 2004;190(2):556-557. doi:10.1016/S0002-9378(03)00773-7

13. Kowalczyk N, Carr Z. Undifferentiated embryonal sarcoma: a case report. Radiol Technol. 2010;81(4):329-334.

14. Jiang Y, Chen F, LeGout J, Alexander L, Stauffer J, Metcalfe A. Undifferentiated embryonal sarcoma of the liver presenting as a hemorrhagic mass in a pregnant woman. Clin Imaging. 2021; 77:58-61. doi:10.1016/j.clinimag.2021.02.037

15. Helling TS, Azoulay D. Ton that Tung's livers. Ann Surg. 2014;259 (6):1245-1252. PubMed PMID: 24335785. doi:10.1097/SLA.000 0000000000370
International Journal of Women's Health

\section{Publish your work in this journal}

The International Journal of Women's Health is an international, peerreviewed open-access journal publishing original research, reports, editorials, reviews and commentaries on all aspects of women's healthcare including gynecology, obstetrics, and breast cancer. The manuscript management system is completely online and includes a very quick and fair peer-review system, which is all easy to use. Visit http://www.dovepress.com/testimonials.php to read real quotes from published authors. 